\title{
Physiological Player Sensing: New Interaction Devices for Video Games
}

\author{
Jérôme Dupire ${ }^{1}$, Viviane $\mathrm{Gal}^{2}$, and Alexandre Topol ${ }^{2}$ \\ ${ }^{1}$ THIM, Universite Paris 8, 2, rue de la Liberté, \\ 93526 Saint Denis, France \\ jerome.dupire@univ-paris8.fr \\ ${ }^{2}$ CEDRIC, CNAM, 292, rue St Martin, \\ 75003 Paris, France \\ \{viviane.gal, alexandre.topol\}@enam.fr
}

\begin{abstract}
In traditional video games, players are used to handle well known devices such as a keyboard or a mouse. In a pervasive game, no virtual world exists, since players evolve in a real environment. Hence, new kinds of interaction devices are needed. The physiological approach presented in this paper is one of the most promising solutions. We have build our own sensing tee-shirt on which physiological sensors are sewed. It makes it possible to monitor in real time the players' physiological states with a minimum of mechanical constraints.
\end{abstract}

Keywords: Wearable Computing, Physiological Sensor, Embedded System, Video Game.

\section{Introduction}

Our work is part of a national funded project named PLUG (PLay Ubiquitous Games). The aim of PLUG is to propose a new way for people to visit a museum. The base idea of this novel approach is to turn agreeing visitors into players and the museum into a game are(n)a.

Each player will be given a mobile platform, such as a mobile phone or a Personal Digital Assistant (PDA). This device makes it possible for the player to receive information from the game server. Traditional interactions between players and the game will be performed through this device, which will be linked to the game server by a Wi-Fi connection (Fig.1). The other technological gaming elements, such as RFID tags or beacons, will be disposed in the museum. According to the game scenario, players will have to interact with them in order to complete various challenges.

In this project, our work package consists in proposing a new kind of interaction in the game environment. The idea is to sense the physiological state of each player and to feed the game engine with these new signals. Hence, for our game, feelings, together with geolocalization, become one of the most important interface between the player and the game, making the usual gestual (through standard gaming devices) and visual interface (through screens) unusable. 
Our ultimate goal is to bring up a precise psycho-emotional state of the player. Although this approach is new in video games, we are however guided by studies relating to emotions. Indeed, psychophysiology tries today to reveal human emotions according to several biometric signals. It also studies the effects of the affect on human physiology [1].

In the next sections of this article, we present the work accomplished until now. It relates mainly to the sensors and their assembly on the player. We also detail the test protocol set up as well as the first results obtained. Lastly, we conclude by explaining the next stages that should transform this preliminary work into a central component of the middleware developed for PLUG.

\section{Hardware Description}

Each player will be equipped with specific sensors. We chose to detect the following physiological variables: the electric activity of the heart $(\mathrm{EKG})$, the muscular electric activity, the skin temperature (ST), the galvanic skin response (GSR), the breathing rate $(\mathrm{Br})$, the blood pressure (BVP) with the method described in [7]. In addition to those sensors, we have associated a tri-axis accelerometer whose purpose is to raise ambiguities when dealing with close physical or physiological states. For example, does the heart rate increase because the player started to run or because he/she is stressed?

The $\mathrm{Br}$ sensor is based on a variable resistor whose value depends on its physical deformation. The electrodes used for the EKG are Red Dot (3M). For the final sensor tee-shirt, they will be replaced by textile surfaces integrated into the sleeves, as in [4]. The temperature sensor is a LM385, sewed so that it is in contact with the skin. The accelerometer is a ADXL330 (from Analog Devices Inc.). The GSR sensor is integrated in a glove worn by the player.

The set of sensors is connected to the ATMEGA168 (ATMEL) microcontrolor of an Arduino board [2]. We chose this particular board for our tests for two main reasons. Firstly, because the GUI and the progamming language close to $\mathrm{C}++$ make it very easy to use. Secondly, because a great community of developers exists around this material and many shared examples and projects can be found.

The link between the Arduino board and the distant data processing system is made by a point-to-point ZigBee radio connection implemented through XBee modules (Maxstream). Here also, this particular material was chosen for its simplicity but also for its already tested use in contexts close to ours, like house automation or health monitoring [3].

The communication between the embedded ZigBee module and the PC is made through a FT232 (FTDI) interface which makes it possible to set a virtual serial port.

All sensors and electronics are sewed on a cloth, making it very easy for the player to literally wear the sensors. The tee-shirt textile is stretchable in order to fit various players' shapes and to ensure that the electrodes are well plated on the skin. Figure 1 shows the various zones reserved for the various sensors on the tee-shirt. The choice of these various zones was made by seeking the best compromise between an ideal placement for collecting strong signals and the least possible constraining or awkward placement for the player. 


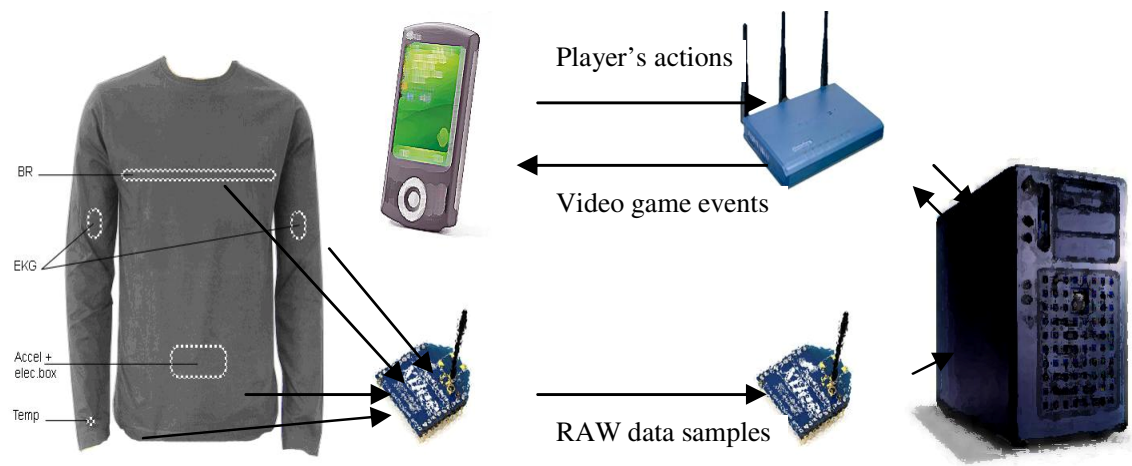

Fig. 1. Position of the various sensors on the tee-shirt, wireless Zigbee and Wifi networks

Moreover, the assembly is made in such a way that wires can slide while arms are moving. A strong constraint of the device is that it must be sufficiently light and discrete to be installed quickly on visitors of the museum. Moreover, it must allow a total liberty of action for the player, without any handicap of weight or handiness. Any exemption from this rule could lead visitors to avoid playing the game or to give up during a game session.

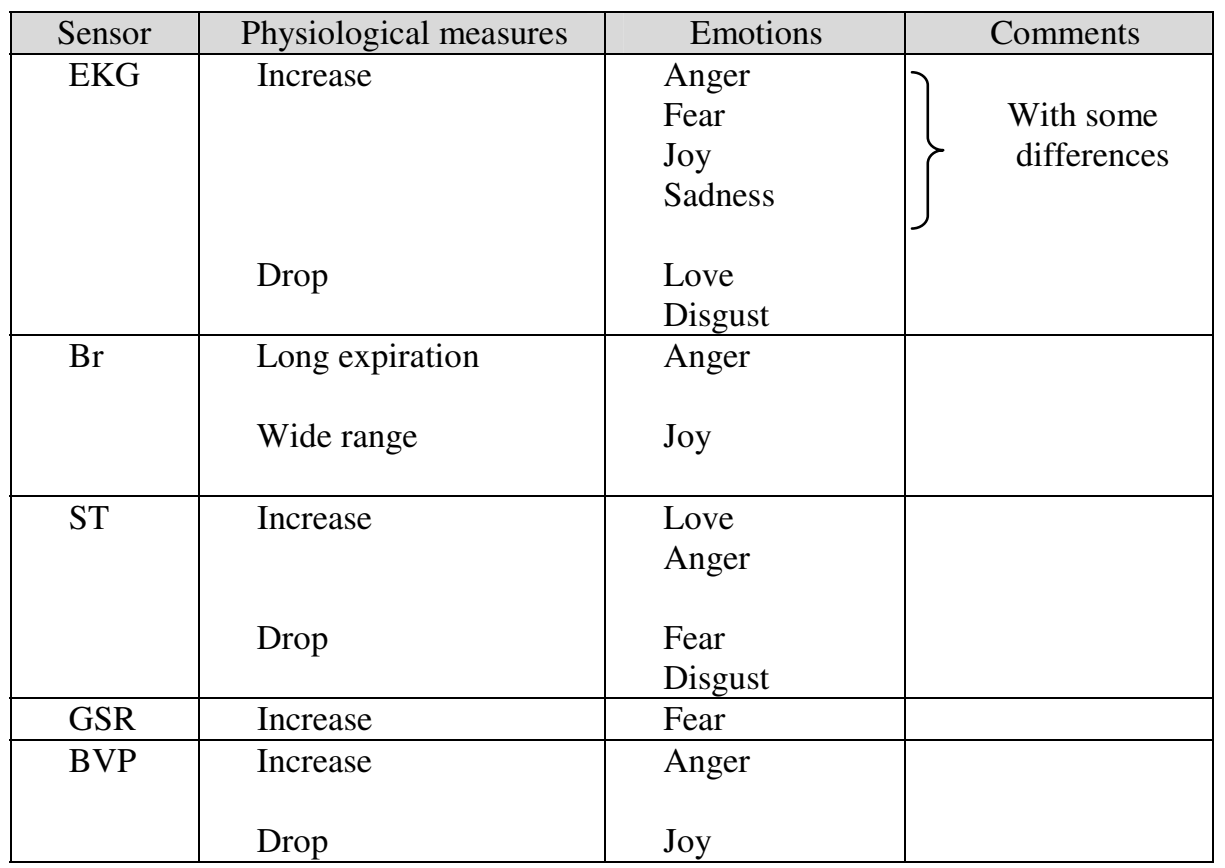

\section{Experimentation}

In the field of psychology, there is a great number of works relating to physiological measurements of psychological phenomena [6], [7], [8]. Our purpose is not to remake 
these experiments. We want to rely on those works and show that our device answers some of the criteria known and validated by the professionals of this field. To do so, we test users in a passive mode. In a second stage to come, the interactivity will be integrated to achieve our goal of emotionnal driven video games. To do so, we will have to build the emotional identity card of the player with which it will be possible to imagine some new game designs.

The following table gives some examples of feelings according to physiological variations [9]. The combination of various measurements make it possible to distinguish the type of emotion. This distinction is reinforced by the accelerometers.

Our test bench is composed by the sensors carried by the user, but also by a camera and a microphone that record the user and finally by a video submitted to the user. The camera and the microphone make it possible for this first phase of tests to put in correspondence the physical behavior (body, verbal,...) and the physiological behavior of the user. The movie watched by the user is an edition carefully timestamped of still images, sounds, movie extracts that are (or not) emotionnally charged. It thus includes calm and key periods.

The user wears the tee-shirt and a mitten on which sensors take place. Measurements are taken remotely thank to the zigbee module. In order to avoid the emotional parasites, the user is isolated and installed comfortably in a room slightly enlightened in front of a screen. The screen includes an almost invisible camera and a microphone. The film is projected during a fixed duration (around 26 minutes).

The perception and emotional reactions being different from one user to the other, we decided to test two groups of adults: a female group and a male group in the same proportion. A set of 10 people currently lends itself to our experimentation. This phase enables us to adjust the device. The results are stored in files and will be analyzed at the end of the process. A real time visualization tool of the sensors signals confirms that the participants reactions seem in conformity with what was awaited. For example, while degusting images were displayed on the screen, the remote visualization tool depicted heart activity and a skin temperature falloffs (Figure 2.). The complete analysis and filtering of the results will be decisive for the choices of the significant elements to retain.

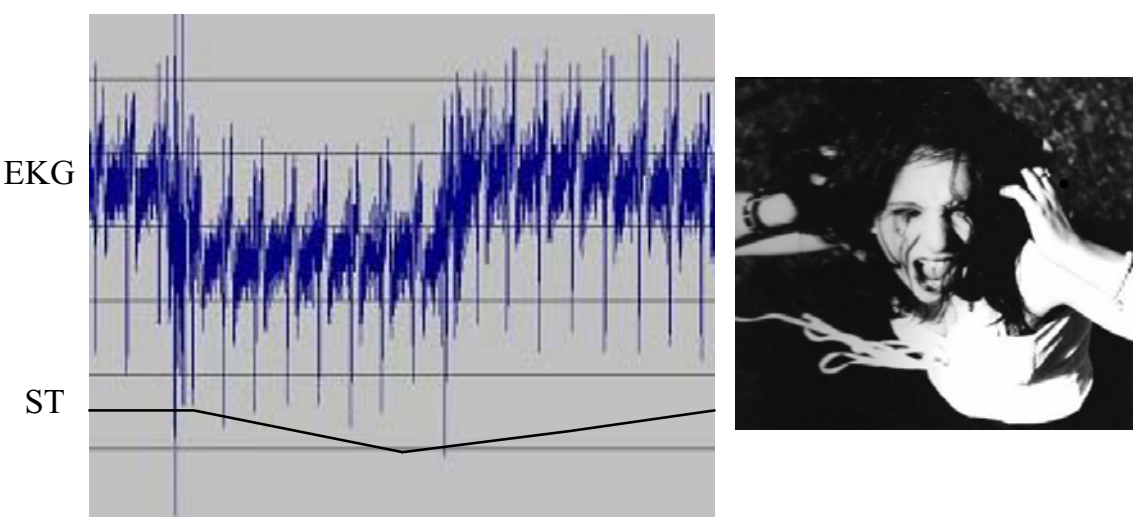

Fig. 2. EKG and ST curves while the right picture is displayed with a scream of terror sound 


\section{Discussion and Future Work}

Taking into account sensors values in a video game can be done in two different ways. The first and most simple one consists in the use of the raw data by the game engine. It will thus modulate in real time the scenario according to values of one or more physiological variables. For example, the aiming cross in a FPS game can shake increasingly when the heart beat or the galvanic skin response are getting to high.

The second manner to deal with signals is to try to interpret the variations of the whole or a part of recorded physiological variables, in order to bring up a more precise psycho-emotional state and send according events to the game server.

This second way of dealing with emotions is much more interesting and is our goal. It would make it possible to provide to the game designer some new tools which could help :

- to conceive and/or validate its traditional game design by finding out if the user really senses the action as it was imagined,

- to develop an adaptive scenario based on the emotions of players,

- to simply take into account the user's emotion like any other traditionnal way of interacting.

First feedbacks from the tests show that all sensors do not work properly during the sessions lengths and should be tuned before a second bunch of test. Some artefacts appear once in a while on curves and may be caused by some interferences between sensors. This problem should also be seriously considered.

Our work will continue with the analysis of signals. Recorded signals should be imported in MathLab in order to ease the determination of the right computations needed to extract feelings. If this step succeeds, we will then code the right filters within our visualization tool to trigger feeling events. The last step before the game designer can truely take into account the player's emotion in its scenario will be to feed the game engine with the feeling events.

Acknowledgments. The authors would like to thank J.F. Lambert (Univ.Paris 8), for his help and welcoming in his psycho-world. The PLUG project is funded by the French national agency for research (ANR).

\section{References}

1. Berntson, G., Caccioppo, J., Tassinary, G.: Handbook of psychophysiology. Cambridge University Press, Cambridge (2007)

2. The Arduino project, http://www . arduino.cc/

3. Dagtas, S., Pekhteryev, G., Sahinoglu, Z.: Multi-Stage Real Time Health Monitoring via ZigBee in Smart Homes. In: Proceedings of AINAW, May 21 - 23, vol. 2 (2007)

4. Finni, T., Hu, M., Kettunen, P., Vilavuo, T., Cheng, S.: Measurement of EMG activity with textile electrodes embedded into clothing. Journal Physiol. Meas. 28, 1405-1419 (2007)

5. Gross, J.J., Levenson, R.W.: Emotion elicitation using films. Cognition and Emotion 9, 87$108(1995)$ 
6. Nasoz, F., Lisetti, C.L., Alvarez, K., Finkelstein, N.: Emotion Recognition from Physiological Signals for User Modeling of Affect. In: Brusilovsky, P., Corbett, A.T., de Rosis, F. (eds.) UM 2003. LNCS, vol. 2702, Springer, Heidelberg (2003)

7. Sorvoja, H.: Noninvasive blood pressure pulse detection and blood pressure determination. Oulu University Press (2006)

8. Villon, O., Lisetti, C.: A User Model of Psycho-Physiological Measure of Emotion. In: Conati, C., McCoy, K., Paliouras, G. (eds.) UM 2007. LNCS (LNAI), vol. 4511, pp. 319 323. Springer, Heidelberg (2007)

9. Scheff, T.J.: A Taxonomy of Emotions: How Do We Begin (2005), http://www.soc.ucsb.edu/faculty/scheff/47.html 